Sādhanā Vol. 40, Part 3, May 2015, pp. 863-873. (C) Indian Academy of Sciences

\title{
Numerical calculation of particle collection efficiency in an electrostatic precipitator
}

\author{
NARENDRA GAJBHIYE ${ }^{1, *}, \mathrm{~V} \mathrm{ESWARAN}^{2}$, A K SAHA $^{1}$ and \\ ANOOP KUMAR ${ }^{3}$ \\ ${ }^{1}$ Department of Mechanical Engineering, IIT Kanpur, Kanpur 208016, India \\ ${ }^{2}$ Department of Mechanical and Aerospace Engineering, IIT Hyderabad, \\ Hyderabad 502205, India \\ ${ }^{3}$ Department of Mechanical Engineering, NIT Hamirpur, Hamirpur 177005, India \\ e-mail: narendra@iitk.ac.in
}

MS received 30 June 2014; revised 30 October 2014; accepted 12 December 2014

\begin{abstract}
The present numerical study involves the finding of the collection efficiency of an electrostatic precipitator (ESP) using a finite volume (ANUPRAVAHA) solver for the Navier-Stokes and continuity equations, along with the Poisson's equation for electric potential and current continuity. The particle movement is simulated using a Lagrangian approach to predict the trajectory of single particles in a fluid as the result of various forces acting on the particle. The ESP model consists of three wires and three collecting plates of combined length of $\mathrm{L}$ placed one after another. The calculations are carried out for a wire-to-plate spacing $H=0.175 \mathrm{~m}$, length of ESP $L=2.210 \mathrm{~m}$ and wire-to-wire spacing of $0.725 \mathrm{~m}$ with radius of wire $R_{\text {wire }}=10$ $\mathrm{mm}$ and inlet air-particle velocity of $1.2 \mathrm{~m} / \mathrm{s}$. Different electrical potentials $(\varphi=15-$ $30 \mathrm{kV}$ ) are applied to the three discharge electrodes wires. It is seen that the particle collection efficiency of the ESP increases with increasing particle diameter, electrical potential and plate length for a given inlet velocity.
\end{abstract}

Keywords. ESP; FVM; EHD; corona discharge.

\section{Introduction}

Electro-hydrodynamics flows (EHD) are those in which fluid motion occurs in the presence of an electrostatic force. Electrostatic precipitators (ESPs) are used in thermal power stations, cement industry, etc., to remove particulate matter from the exhaust gases of furnaces before releasing those gases to the atmosphere. In ESPs, harmful soot particles are removed from flue gases by electrostatic forces that drive these particles onto collecting plates so that they can be removed. The collection efficiency, which is the fraction of the particles collected, is an important parameter for determining the performance of the ESP and hence needs to be calculated. Optimal ESP

${ }^{*}$ For correspondence 
design requires accurate prediction of the collection efficiency, which in turn requires accurate computation of fluid mechanics and particle transport parameters.

Theoretical and numerical research has been previously done on ESPs to predict the efficiency (Al- Hamouz \& Abuzaid 2002; Cooperman 1971; Kocik et al 2005). In 1999, Kim \& Lee (1999) did an experimental study and compared the results with theoretical models. The authors investigated the optimized parameters for an ESP, including the discharge wire diameter, wire-to-wire spacing, wire-to-plate spacing, air flow velocity, as well as turbulence, and applied wire voltage.

Lagrangian simulations of particle transport in wire-plate ESP were performed by several researchers (Choi \& Fletcher 1998; Gallimberti 1998; Nikas et al 2005). These simulations are based on particle tracking in the flow field calculated by a finite volume solution of the NavierStokes equations, including a turbulence k- $\varepsilon$ model and the influence of electrostatic forces. The coupling between the fluid flow and electric field has examined by Schmid \& Vogel (2003). In this model, a comparison between the Eulerian and Lagrangian approaches was made, showing the Lagrangian approach as superior. Akana (2006) \& Shitole (2007) did a parametric study of the performance of an ESP for different wire plate spacings, wire voltages and particle diameters.

\section{Problem definition}

In an ESP, there are two collecting plates with a high-voltage wire (discharge electrode) placed between them, so that the particle-laden air passes between the plates and across the discharge electrode. The latter ionizes the air stream and transfers electric charge to particles which are then driven by electrostatic forces to the collecting plates on which they are deposited, and later removed. The collecting plates of ESP are often placed in tandem, one after another, to increase the total collection efficiency, as particles missed by the first may be collected by the second, and so on. In the present study, 2D simulations are done for a three-wire combination placed inbetween collecting plates with a combined length $\mathrm{L}$, as shown in figure 1 . In the present problem, the ESP model is analyzed with wire-plate spacing $\mathrm{H}$ of $0.1750 \mathrm{~m}$, wire-wire spacing $0.725 \mathrm{~m}$, and inlet and outlet lengths, to and from the first and third discharge electrodes, of $0.4 \mathrm{~m}$ and $0.36 \mathrm{~m}$, respectively. The wire potentials range between $15.0 \mathrm{kV}$ and $30.0 \mathrm{kV}$.

The simulations are done for the upper half-geometry, because of the symmetric nature of the geometry and the mean flow. No-slip boundary conditions are applied on the top boundary, which is the wall, while symmetry BCs are used at the bottom boundary. The left boundary is the inlet, where the inlet velocity is specified, while the right boundary is the outlet where homogeneous Neumann outflow conditions are specified for the flow variables, except pressure which is taken to be homogeneous Dirichlet. The simulation parameters used for the present case are shown in table 1.

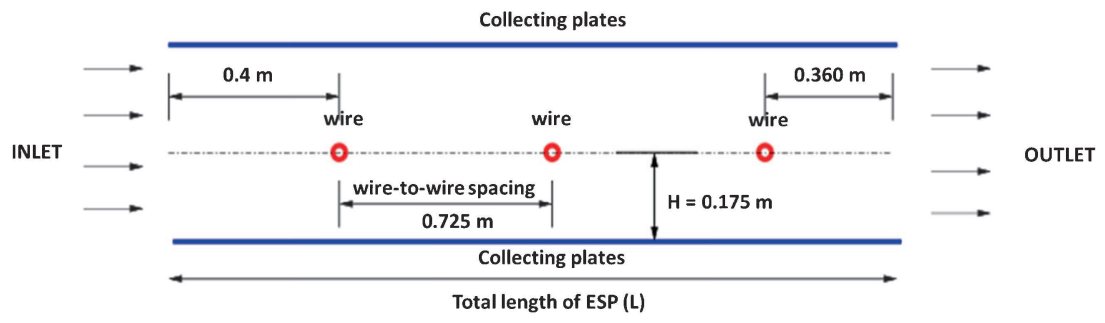

Figure 1. Schematic of electrostatic precipitator. 
Table 1. Parameters are used for simulations.

\begin{tabular}{ll}
\hline Radius of wire, $R_{\text {wire }}$ & $10 \mathrm{~mm}$ \\
Length of the ESP, $L$ & $2.210 \mathrm{~m}$ \\
Wire-plate spacing, $H$ & $0.175 \mathrm{~m}$ \\
Permittivity, $\varepsilon$ & $8.854 \times 10^{-12} \mathrm{~F} / \mathrm{m}$ \\
Ion mobility rate, $b$ & $1.6 \times 10^{-4} \mathrm{~m}^{2} / \mathrm{Vs}$ \\
Charge diffusion coefficient, $D$ & $1.0 \times 10^{-2} \mathrm{~m}^{2} / \mathrm{s}$ \\
Relative permittivity of the medium, $\varepsilon_{\mathrm{r}}$ & 1.0 \\
Molecular viscosity of gas, $\mu$ & $9.3 \times 10^{-4} \mathrm{~kg} / \mathrm{m} \mathrm{s}$ \\
Gas velocity at inlet, $u$ & $1.2 \mathrm{~m} / \mathrm{s}$ \\
\hline
\end{tabular}

\section{Governing equations}

The governing equations for the flow problem are the continuity and Navier-Stokes equation with an additional term take into account the effect of the electric field on the flow field. In addition, the Poisson equation for the electric potential, $\varphi$, and a convective-diffusion equation for the electric charge density, $\rho_{\text {ions }}$, are also solved. The effect of the particles on the electric and velocity fields is small, and a useful simplification is made by assuming one-way coupling, i.e., the electric field affects the particle motion, but not vice-versa.

The unsteady governing equations for incompressible electro-hydrodynamic flows in two dimensional, including the electrostatic force as a source term, are given as

Continuity equation

$$
\frac{\partial u}{\partial x}+\frac{\partial v}{\partial y}=0
$$

$X$-momentum equation

$$
\frac{\partial u}{\partial t}+u \frac{\partial u}{\partial x}+v \frac{\partial u}{\partial y}=-\frac{1}{\rho} \frac{\partial p}{\partial x}+\frac{\mu}{\rho}\left(\frac{\partial^{2} u}{\partial x^{2}}+\frac{\partial^{2} u}{\partial y^{2}}\right)+\frac{\rho_{\text {ions }}}{\rho} E_{x}
$$

Y-momentum equation

$$
\frac{\partial v}{\partial t}+u \frac{\partial v}{\partial x}+v \frac{\partial v}{\partial y}=-\frac{1}{\rho} \frac{\partial p}{\partial y}+\frac{\mu}{\rho}\left(\frac{\partial^{2} v}{\partial x^{2}}+\frac{\partial^{2} v}{\partial y^{2}}\right)+\frac{\rho_{i o n s}}{\rho} E_{y}
$$

where $E_{x}=-\frac{\partial \varphi}{\partial x}$ and $E_{y}=-\frac{\partial \varphi}{\partial y}$ are the electric field components along $x$ and $y$ directions, $\varphi$ is the electric potential field and $\rho_{\text {ions }}$ is the ion charge density.

\section{Governing equations for electric field}

Under the assumption of a monopolar corona (positive, same polarity of that of discharge electrode with the charge transfers from particles to ions being neglected) we arrive at the following governing equations (Medlin et al 1998) describing ionic charge densities and the resulting electric fields:

$$
\begin{gathered}
\nabla \cdot \vec{E}=-\frac{\rho_{\text {ions }}}{\varepsilon} \\
\vec{E}=-\nabla \varphi \\
\vec{J}=\rho_{\text {ions }} b \vec{E}-D \nabla \rho_{\text {ions }} \\
\frac{\partial \rho_{\text {ions }}}{\partial t}+\nabla \cdot \vec{J}=0
\end{gathered}
$$


where $\rho_{\text {ions }}$ is the ion charge density, $b$ is the ion mobility, $\varepsilon$ is the free space permittivity and $D$ is the diffusion coefficient for the charge density. Combining the above, the following two equations are solved with the false transient formulation to get the electrical potential and the ion charge density:

$$
\begin{gathered}
\nabla^{2} \varphi=\frac{\rho_{\text {ions }}}{\varepsilon} \\
\frac{\partial \rho_{\text {ions }}}{\partial t}+\mathrm{b} \nabla \cdot\left(\rho_{\text {ions }} \vec{u}\right)=D \nabla^{2} \rho_{\text {ions }}
\end{gathered}
$$

\subsection{Boundary conditions}

The boundary conditions for velocity components, pressure, electric potential and ion charge density at the wire and grounded plate are given as

Wire: $u=v=0$

$$
\begin{gathered}
\frac{\partial p}{\partial n}=0 \\
\varphi=\varphi_{\text {applied }} \\
\rho_{\text {ions }}=\rho_{\text {wire }}
\end{gathered}
$$

Plate: $u=v=0$

$$
\begin{gathered}
\frac{\partial p}{\partial n}=0 \\
\varphi=0 \\
\frac{\partial \rho_{i o n s}}{\partial n}=0
\end{gathered}
$$

while at the inlet and outlet boundaries they are

Inlet: $u=1.2 \mathrm{~m} / \mathrm{s}, v=0$

$$
\begin{gathered}
\frac{\partial p}{\partial n}=0 \\
\frac{\partial \varphi}{\partial n}=0 \\
\frac{\partial \rho_{i o n s}}{\partial n}=0
\end{gathered}
$$

Outlet: $\frac{\partial u}{\partial n}=\frac{\partial v}{\partial n}=0$

$$
\begin{gathered}
p=0 \\
\frac{\partial \varphi}{\partial n}=0 \\
\frac{\partial \rho_{\text {ions }}}{\partial n}=0
\end{gathered}
$$

The quantity $\varphi_{\text {applied }}$ is the voltage being applied to the wires, while $\rho_{\text {wire }}$ is the charge density at the wires. The value of $\rho_{\text {wire }}$ is unknown and hence cannot be supplied directly as boundary 
condition. It is found by iterations, by requiring that at the surface of the wire the electric field equals the value given by the modified Peek's formula (Medlin et al 1998)

$$
E_{\text {Peek }}=A \delta+B \sqrt{\frac{\delta}{R_{\text {wire }}}}
$$

where $A=32.3 \times 10^{5} \mathrm{~V} / \mathrm{m}$ and $B=0.846 \times 10^{5} \mathrm{~V} / \mathrm{m}^{1 / 2}$ for air, $R_{\text {wire }}$ the radius of wire in $\mathrm{m}$, and $\delta$ is the density of gas relative to gas at standard pressure and temperature. The value that results in the correct electric field at the wire surface is taken to be the correct value of $\rho_{\text {wire }}$.

\section{Equation for particle motion}

Once the steady-state velocity and electric field are obtained by the false transient timestepping of the flow and electric field equations, the particle trajectories can be computed. The Lagarangian approach is used to predict the trajectory of single particles in the fluid flow as a result of drag and electrostatic forces. Neglecting the gravity force, the equations for particle motion are reduced to

$$
\begin{aligned}
& \frac{d u_{p}}{d t}=\frac{3 \rho C_{d}}{4 d_{p} \rho_{p}}\left(u-u_{p}\right)\left|\vec{u}-\vec{u}_{p}\right|+\frac{q_{p} E_{x}}{m_{p}} \\
& \frac{d v_{p}}{d t}=\frac{3 \rho C_{d}}{4 d_{p} \rho_{p}}\left(v-v_{p}\right)\left|\vec{u}-\vec{u}_{p}\right|+\frac{q_{p} E_{y}}{m_{p}}
\end{aligned}
$$

where $\vec{u}$ and $\vec{u}_{p}$ are the velocity of fluid and particle while $(u, v)$, and $\left(u_{p}, v_{p}\right)$ are their $x$ and $y$ components, $q_{p}$ is the charge on the particle, $\rho_{p}$ is the density of the particle, $E_{x}$ and $E_{y}$ are $x$ and $y$-direction electric field intensity.

The drag coefficient of spherical particles is given by

$$
C_{d}= \begin{cases}\frac{24}{R e_{p}}\left(1+0.1667 R e_{p}^{0.667}\right) & \text { for } R e_{p}<1000 \\ 0.44 & \text { for } R e_{p}>1000\end{cases}
$$

The particle Reynolds number is

$$
R e_{p}=\frac{\rho\left|\vec{u}-\vec{u}_{p}\right| d_{p}}{\mu}
$$

The charge on a particle is determined by the formula

$$
q_{p}=\frac{3 \varepsilon_{r}}{\varepsilon_{r}+2} \pi \varepsilon d_{p}^{2}|E|
$$

Here $d_{p}$ is the diameter of the particle, $\varepsilon_{r}$ is the relative permittivity of the particle, $\varepsilon$ is permittivity of free space, and $E\left(=\sqrt{E_{x}^{2}+E_{y}^{2}}\right)$ is the local electric field.

\section{Results and discussions}

In the present study, the calculations are carried out for a wire plate spacing, $H=0.175 \mathrm{~m}$, length of ESP $L=2.210 \mathrm{~m}$ and wire to wire spacing of $0.725 \mathrm{~m}$ with radius of wire, $R_{\text {wire }}=10 \mathrm{~mm}$. 
The inlet length to the first wire and the outlet length after the last wire are $0.4 \mathrm{~m}$ and $0.36 \mathrm{~m}$ respectively. Uniform velocity is specified at the inlet of magnitude $1.2 \mathrm{~m} / \mathrm{s}$. Different electrical potentials are applied to the wires of (1) $15 \mathrm{kV}$ (2) $20 \mathrm{kV}$ (3) $25 \mathrm{kV}$ and (4) $30 \mathrm{kV}$, for the cases being studied. The effect of the smooth collecting plates (in industrial applications these plates may be corrugated) on the collecting efficiency of ESP is analyzed, for the parameters given in table 1.

The computational mesh contains 19,482 cells with 70 points on the wire (shown for a single wire in figure 2). Near the wire, the mesh is highly refined because of the large gradients of the electric potential expected there. The grid is generated using the commercial GridZ software, with an exponential function for clustering the near-wire region.

The collection efficiency can be defined as the ratio of the number of particles collected by the collector plates to the total number of particles entering at the inlet. Because of the oneway coupling assumption made and also the assumption that the particles do not interact, the collection efficiency is independent of the particle density, in these calculations. To determine the collection efficiency for each case, 100 particles are tracked in the flow field from the inlet of the ESP. These particles are assumed to be uniformly distributed and released with the same velocity as the air-stream from the inlet boundary. Any particle striking the collecting plate is assumed to be collected by the plate. The collection efficiency $(\eta)$ is determined as follows:

$$
\eta=\left(1-\frac{N_{\text {out }}}{N_{\text {in }}}\right) \times 100
$$

where $N_{\text {out }}$ is the number of particles leaving the exit and $N_{\text {in }}$ is the number of the particles entering at the inlet.

The effect of wire electrical potential and the diameter of particles on the trajectory of the particles are presented here. Figure 3 shows the particle paths of ten particles, uniformly placed at the inlet, when an electrical potential of $15 \mathrm{kV}$ is applied at the wire, for different particle diameters of 2, 3, 4 and $5 \mu \mathrm{m}$.

From the figure it is observed that the particles deviate from their previous path as they approach the wire locations. The path lines start deviating from the first wire and this deviation increases as the particle successively passes the second and third wires. The particles that strike the wall are "collected". However, not all of them get collected at the grounded plate, as some of them escape through the flow exit. The flow field free ions transfer their charge to the particles

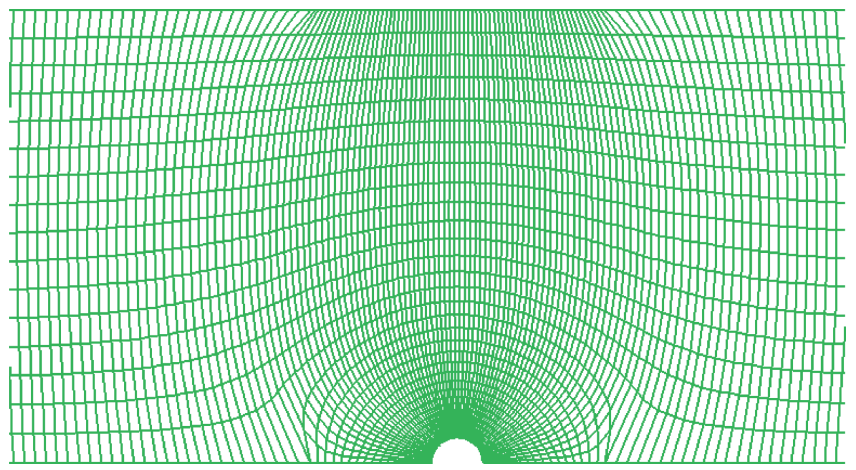

Figure 2. Computational mesh. 

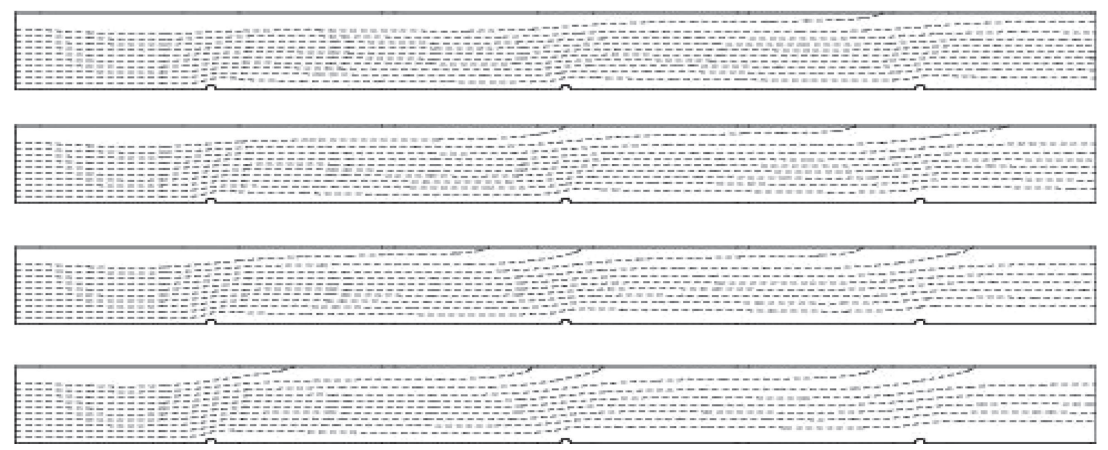

Figure 3. Particle path for electrical potential $\varphi=15 \mathrm{kV}$, wire-plate spacing $=0.1750 \mathrm{~m}$ and particle diameters of (1) $\mathrm{dp}=2.0 \mu \mathrm{m}$; (2) $\mathrm{dp}=3.0 \mu \mathrm{m}$; (3) $\mathrm{dp}=4.0 \mu \mathrm{m}$; (4) $\mathrm{dp}=5.0 \mu \mathrm{m}$.

which get charged. Particle deviation is due to effect of the electrical field on the charged particles. When a charged particle reaches the collecting plate, the charge on the particle is assumed to be passed on to the collecting plate. From the equation of motion of the particle the drag force is inversely related to the square of particle diameter while the electrostatic force is inversely related to the particle diameter. Therefore the electrostatic force/Drag force ratio increases with increasing particle diameter and the collection efficiency is found to be higher for higher particle diameters. From figure 3, it is evident that the particles having higher diameter are collected sooner. Therefore with increase in the diameter of particles the collection efficiency increases. The collecting efficiency for $5 \mu \mathrm{m}$ particles for wire potential $15 \mathrm{kV}$ is found to be $48 \%$ whereas for $2 \mu \mathrm{m}$ particles it is $16 \%$.

Figure 4, figure 5, and figure 6 similarly show the path lines for different particle diameters of $2,3,4$, and $5 \mu \mathrm{m}$ for wire potentials of $20 \mathrm{kV}, 25 \mathrm{kV}$, and $30 \mathrm{kV}$ respectively. It is observed that as the electrical potential increases, collection efficiency increases, because higher voltage results in higher particle charge $q_{p}$ and consequently more particles get collected. In fact, for the particles of higher diameters at higher wire potentials, all the particles are collected by the plate, and their collection efficiency is $100 \%$.

Figure 7 shows the collection performance for different particle diameters at different electrical potentials of $\varphi_{\text {applied }}=15,20,25$ and $30 \mathrm{kV}$. From the figure it is observed that the effect

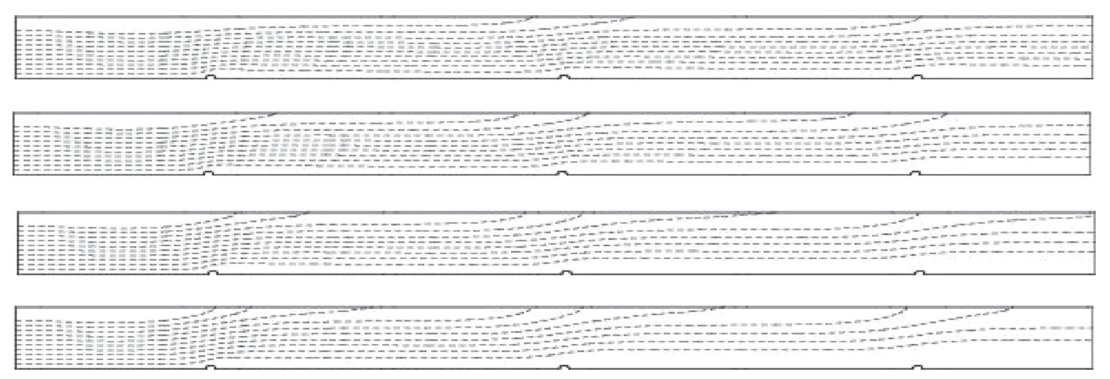

Figure 4. Particle path for electrical potential $\varphi=20 \mathrm{kV}$, wire-plate spacing $=0.1750 \mathrm{~m}$ and particle diameters of (1) $\mathrm{dp}=2.0 \mu \mathrm{m}$; (2) $\mathrm{dp}=3.0 \mu \mathrm{m}$; (3) $\mathrm{dp}=4.0 \mu \mathrm{m}$; (4) $\mathrm{dp}=5.0 \mu \mathrm{m}$. 

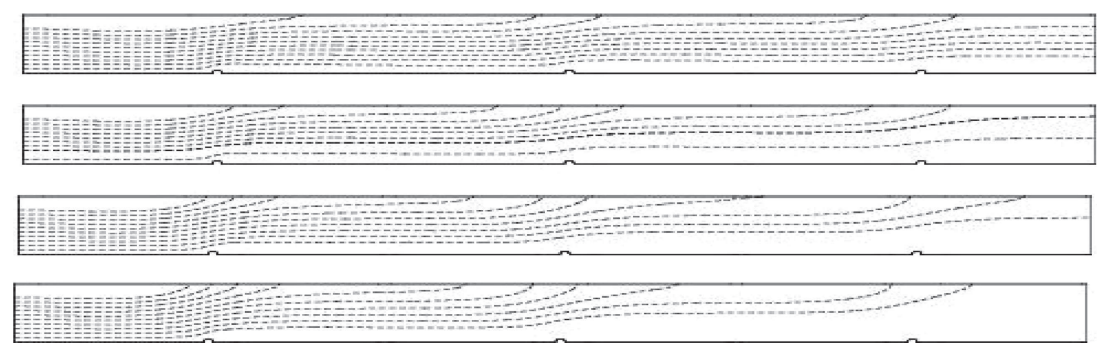

Figure 5. Particle path for electrical potential $\varphi=25 \mathrm{kV}$, wire-plate spacing $=0.1750 \mathrm{~m}$ and particle diameters of (1) $\mathrm{dp}=2.0 \mu \mathrm{m}$; (2) $\mathrm{dp}=3.0 \mu \mathrm{m}$; (3) $\mathrm{dp}=4.0 \mu \mathrm{m}$; (4) $\mathrm{dp}=5.0 \mu \mathrm{m}$.
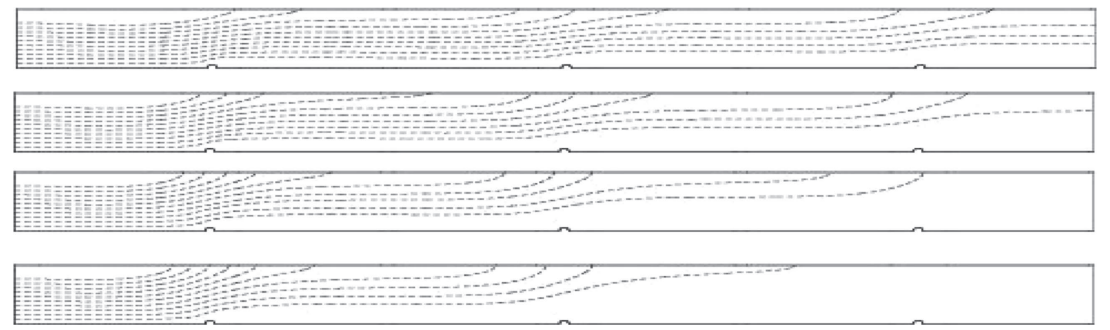

Figure 6. Particle path for electrical potential $\varphi=30 \mathrm{kV}$, wire-plate spacing $=0.1750 \mathrm{~m}$ and particle diameters of (1) $\mathrm{dp}=2.0 \mu \mathrm{m}$; (2) $\mathrm{dp}=3.0 \mu \mathrm{m}$; (3) $\mathrm{dp}=4.0 \mu \mathrm{m}$; (4) $\mathrm{dp}=5.0 \mu \mathrm{m}$.

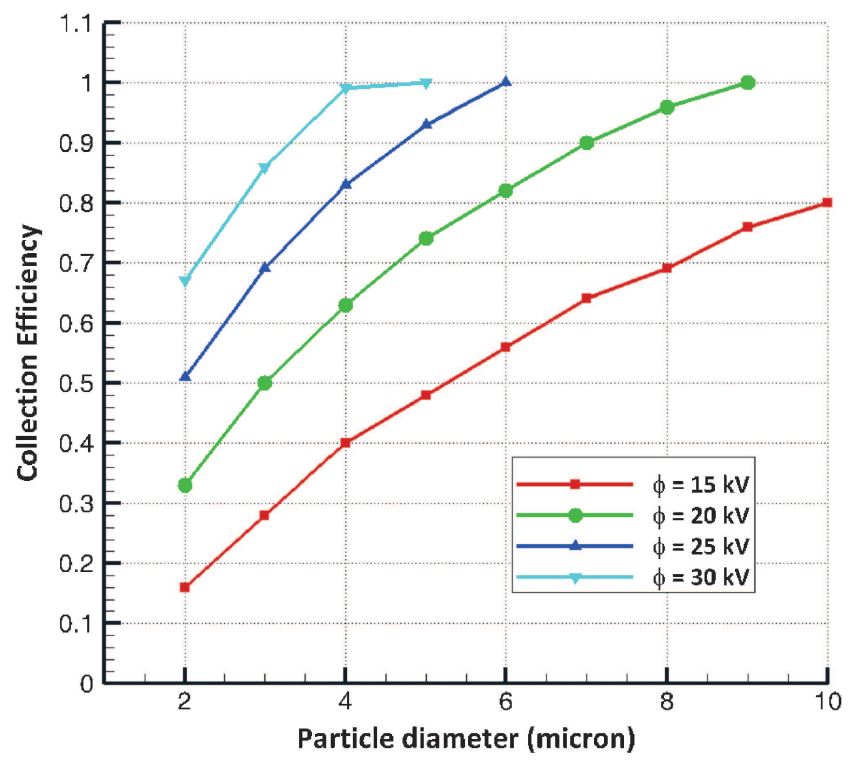

Figure 7. Variation of collection efficiency with the particle diameter at different wire potentials of (1) $\varphi=15 \mathrm{kV}$; (2) $\varphi=20 \mathrm{kV}$; (3) $\varphi=25 \mathrm{kV}$; (4) $\varphi=30 \mathrm{kV}$. 
of particle diameter on collection performance is very strong, and as the diameter of the particle increases, the collection efficiency of the ESP increases. The larger diameter particles are collected very soon for higher wire potentials and the collection efficiency reaches $100 \%$.

However, this is not the case for the smaller diameter particles, such as $2 \mu \mathrm{m}$ where collection efficiency is found to be only $33 \%$ for $\varphi_{\text {applied }}=20 \mathrm{kV}$. Medium size particles $(5 \mu \mathrm{m})$ are better collected, and the collection efficiency for this particle size is $74 \%$. It is also observed that as the wire potential increases, collection efficiency is increased for a fixed particle size. An increase in wire potential from $20 \mathrm{kV}$ to $25 \mathrm{kV}$ enhances the collection efficiency by $25 \%$ for particles of diameter $5 \mu \mathrm{m}$. For a wire potential of $20 \mathrm{kV}$ all particles of diameter more than $8.0 \mu \mathrm{m}$ get picked up by the collector plates and the collection efficiency is $100 \%$. The collection efficiency is also $100 \%$ for particles of size more than 4 and $5 \mu \mathrm{m}$ respectively, for the wire potential of 30 $\mathrm{kV}$ and $25 \mathrm{kV}$.

\section{Mathematical model for predicting the collecting efficiency}

In this section, we present a simple mathematical model developed for predicting the collection efficiency of the ESP for multi-wire geometries from the efficiency of a lower wire configuration. We have validated the model by its predictions with the present simulation results. We propose that the combined efficiency $\eta_{N}$ of an ESP created by concatenating $\mathrm{N}$ single-wire ESPs can be obtained from the one-wire efficiency, $\eta_{1}$, by

$$
\eta_{N}=1-\left(1-\eta_{1}\right)^{N}
$$

where

$\eta_{N}=$ collecting efficiency with $\mathrm{N}$ wires

$\eta_{1}=$ collection efficiency of a single wire configuration

This formula is based on the assumption that the probability of not being collected by each of the concatenated ESPs is constant. If given the collection efficiency for a multi-wire configuration, we can find value of $\eta_{1}$ using Eq. (14), which can then be used to calculate the collection efficiency of other multi-wire configurations. For example, from the efficiency of the two-wire configuration $\eta_{2}$, we get $\eta_{1}=1-\left(1-\eta_{2}\right)^{\frac{1}{2}}$, which can then be used in Eq. 14 . Table 2 shows the collection efficiency of three-wire and six-wire configurations for different particle diameters at an electrical potential of $25 \mathrm{kV}$, thus calculated from $\eta_{2}$. It is seen from the table that the collection efficiency obtained by the mathematical model is within $10 \%$ for the

Table 2. Comparison between the collection efficiencies obtained from the mathematical model (Eq. 14) and numerical simulation for different particle diameters and wire configurations.

\begin{tabular}{lcccc}
\hline Case & Particle diameter (microns) & Computation & Eq. 14 & $\%$ of error \\
\hline Six-wire geometry & 2 & 84 & 71 & 15 \\
& 3 & 100 & 88 & 12 \\
Three-wire geometry & 4 & 100 & 95 & 5 \\
& 2 & 51 & 46 & 9.8 \\
& 3 & 69 & 66 & 4.34 \\
& 4 & 83 & 78 & 6 \\
\hline
\end{tabular}


Table 3. Comparison between the collection efficiencies obtained from the mathematical model (Eq. 14) and numerical simulation with $\eta_{1}$ calculated using the three-wire configuration.

\begin{tabular}{lcccc}
\hline Case & Particle diameter (microns) & Computation & Eq. 14 & \% of error \\
\hline Six-wire geometry & 2 & 84 & 76 & 9.5 \\
& 3 & 100 & 90 & 10 \\
& 4 & 100 & 98 & 2 \\
\hline
\end{tabular}

three-wire configuration and $15 \%$ for the six-wire one. For a better prediction of the collection efficiency of the six-wire configuration, $\eta_{1}$ can be obtained from the three-wire configuration as $\eta_{1}=1-\left(1-\eta_{3}\right)^{\frac{1}{3}}$, which is used to predict the six-wire configuration collection efficiency in table 3, which shows improved results.

\section{Conclusions}

The efficiency of an ESP is found to be strongly determined by the particle diameter. The collection efficiency increases, for a given inlet velocity, with particle diameter and wire potential. For the configuration studied, particles of diameter more than 8,5 and $4 \mu \mathrm{m}$ get picked up by the collector plates (i.e., the collection efficiency is $100 \%$ ) for wire potentials of 20,25 , and $30 \mathrm{kV}$. A mathematical model proposed for predicting the collection efficiency gives reasonable results for multi-wire geometries for different particle diameters.

\section{Nomenclature}

$R_{\text {wire }}$ Radius of wire

$\varepsilon \quad$ Permittivity of free space

$b \quad$ Ion mobility rate

$D \quad$ Charge diffusion coefficient

$\varepsilon_{r} \quad$ Relative permittivity of the medium

$\rho_{\text {part }}$ Density of the particles

$\rho_{\text {ions }}$ Density of the ions

$\mu \quad$ Molecular viscosity of gas

$u, v \quad$ Velocity components

$x, y$ Coordinate of the system

$L \quad$ Total length of the collecting plate

$\vec{J} \quad$ Current density

$\varphi \quad$ Electrical potential

$E_{x} \quad \mathrm{X}$-components of electric field

$E_{y} \quad$ Y-components of electric field

$d_{p} \quad$ Particle diameter

\section{References}

Akana V R 2006 Numerical simulation of electrically charged fluid flows, Thesis for the Degree of Master of Technology, Indian Institute of Technology Kanpur 
Al- Hamouz Z M and Abuzaid N S 2002 Numerical computation of collection efficiency in wire duct electrostatic precipitator. IEEE 2: 1390-1396

Choi B S and Fletcher C A J 1998 Turbulent particle dispersion in an electrostatic precipitator. J. Appl. Math. Model. 22: 1009-1021

Cooperman P 1971 A new theory of precipitator efficiency. Atmos. Environ. 5: 541-551

Gallimberti I 1998 Recent advancements in the physical modeling of electrostatic precipitators. $J$. Electrostat. 43: 219-247

Kallio G A and Stock D E 1992 Interaction of electrostatic and fluid dynamic fields in wire-plate electrostatic precipitators. J. Fluid Mech. 240: 133-166

Kim S H and Lee K W 1999 Experimental study of electrostatic precipitator performance and comparison with existing theoretical prediction models. J. Electrostat. 48: 3-25

Kocik M, Dekowski J and Mizeraczyk J 2005 Particle precipitation efficiency in an electrostatic precipitator. J. Electrostat. 63: 761-766

Medlin A J, Fletcher C A J and Morrow R 1998 A pseudotransient approach to steady state solution of electric field-space charge coupled problem. J. Electrostat. 43: 39-60

Nikas K S P, Varonos A A and Bergeles G S 2005 Numerical simulation of the flow and the collection mechanism inside a laboratory scale electrostatic precipitator. J. Electrostat. 63: 423-443

Schmid H J and Vogel L 2003 On the modeling of the particle dynamics in electro-hydrodynamic flowfields: I. Comparison of Eulerian and Lagrangian modeling approach. Powder Technol. 136: 118-135

Som S K and Biswas G 2000 Introduction to fluid mechanics and fluid machines. Tata McGraw-Hill

Shitole V 2007 Numerical simulation of fly ash and fluid flow in an electrostatic precipitator.Thesis for the Degree of Master of Technology, Indian Institute of Technology Kanpur

White H J 1963 Industrial electrostatic precipitations. Addison-Wesley 